\title{
Isolated Spin Qubits in SiC with a High-Fidelity Infrared Spin-to-Photon Interface
}

\author{
David J. Christle, ${ }^{1}$ Paul V. Klimov, ${ }^{1}$ Charles F. de las Casas, ${ }^{1}$ Krisztián Szász, ${ }^{2}$ Viktor Ivády, ${ }^{2,3}$ \\ Valdas Jokubavicius, ${ }^{3}$ Jawad Ul Hassan, ${ }^{3}$ Mikael Syväjärvi, ${ }^{3}$ William F. Koehl, ${ }^{1}$ Takeshi Ohshima, ${ }^{4}$ \\ Nguyen T. Son, ${ }^{3}$ Erik Janzén, ${ }^{3}$ Ádám Gali, ${ }^{2,5}$ and David D. Awschalom ${ }^{1, *}$ \\ ${ }^{1}$ Institute for Molecular Engineering, University of Chicago, Chicago, Illinois 60637, USA \\ ${ }^{2}$ Wigner Research Centre for Physics, Institute for Solid State Physics and Optics, \\ Hungarian Academy of Sciences, PO Box 49, H-1525, Budapest, Hungary \\ ${ }^{3}$ Department of Physics, Chemistry and Biology, Linköping University, SE-581 83 Linköping, Sweden \\ ${ }^{4}$ National Institutes for Quantum and Radiological Science and Technology, \\ 1233 Watanuki, Takasaki, Gunma 370-1292, Japan \\ ${ }^{5}$ Department of Atomic Physics, Budapest University of Technology and Economics, \\ Budafoki út 8., H-1111, Budapest, Hungary
}

(Received 20 February 2017; revised manuscript received 26 April 2017; published 23 June 2017)

\begin{abstract}
The divacancies in $\mathrm{SiC}$ are a family of paramagnetic defects that show promise for quantum communication technologies due to their long-lived electron spin coherence and their optical addressability at near-telecom wavelengths. Nonetheless, a high-fidelity spin-photon interface, which is a crucial prerequisite for such technologies, has not yet been demonstrated. Here, we demonstrate that such an interface exists in isolated divacancies in epitaxial films of $3 \mathrm{C}-\mathrm{SiC}$ and $4 \mathrm{H}-\mathrm{SiC}$. Our data show that divacancies in $4 \mathrm{H}-\mathrm{SiC}$ have minimal undesirable spin mixing, and that the optical linewidths in our current sample are already similar to those of recent remote entanglement demonstrations in other systems. Moreover, we find that $3 \mathrm{C}-\mathrm{SiC}$ divacancies have a millisecond Hahn-echo spin coherence time, which is among the longest measured in a naturally isotopic solid. The presence of defects with these properties in a commercial semiconductor that can be heteroepitaxially grown as a thin film on $\mathrm{Si}$ shows promise for future quantum networks based on $\mathrm{SiC}$ defects.
\end{abstract}

DOI: 10.1103/PhysRevX.7.021046

\section{INTRODUCTION}

Deep-level defects in semiconductors are attractive systems for exploring quantum information and quantummetrology applications. Similar to atoms, defects can possess electronic spin states with long coherence times that can be initialized and detected optically, but they also have the advantage of being fixed within a solid. The nitrogen-vacancy (NV) center in diamond is the most prominent deep-level defect for advanced quantum optical experiments, and has a rare combination of single-defect addressability, long spin coherence times, and a good optical interface. These properties have been established over five decades of research, and are at the foundation of cutting-edge demonstrations of long-distance photonmediated entanglement, teleportation, and the loopholefree test of Bell's inequalities [1-3].

\footnotetext{
*awsch@uchicago.edu
}

Published by the American Physical Society under the terms of the Creative Commons Attribution 4.0 International license. Further distribution of this work must maintain attribution to the author(s) and the published article's title, journal citation, and DOI.
Subject Areas: Quantum Information,

Semiconductor Physics, Spintronics

In recent years, attention has increasingly been directed towards finding optically active defects with quantum mechanical properties that would complement or improve on those seen in NV centers. In particular, the NV's visiblewavelength emission and the difficultly of growing and microfabricating diamond are significant barriers to longdistance entanglement and creating scalable, integrated nanophotonic structures. Silicon vacancies and nickel centers in diamond [4,5], rare-earth and transition metal ions [6-8], and several different vacancy-related complexes in silicon carbide [9-14] are all examples of possible alternatives that are being explored. These defects can have certain attractive features, but in many cases they have been controlled only in ensembles, have relatively short spin coherence times, or suffer from low initialization and readout fidelities. Even with compelling fundamental or materials advantages, it is difficult to assess a defect's potential role in future quantum technologies until the key milestones that overcome these challenges are reached.

In this work, we meet three of these milestones for divacancy defects in silicon carbide $(\mathrm{SiC})$-we isolate single defects in the cubic 3C polytype of $\mathrm{SiC}$ for the first time, demonstrate that their spin-coherence times are long, and reveal a robust spin-to-photon interface in both $3 \mathrm{C}$ - and 
4H-SiC divacancies that can be used for high-fidelity preparation and readout using resonant infrared light. Each of these is a vital component for entangling remote defects, and establishing them makes a compelling case for utilizing the distinct advantages of $\mathrm{SiC}$ divacancies for long-distance entanglement and quantum networks. An exciting result is that its optical fine structure is akin to that of the diamond NV center, which means that the high-fidelity preparation and readout protocols used there will translate, so long as certain technical figures of merit are similar. In our experiments in $4 \mathrm{H}-\mathrm{SiC}$, we find optical transition linewidths near those in state-of-the-art NV experiments and the existence of highly spin-selective optical transitions useful for high-fidelity spin readout.

Divacancy defects in $\mathrm{SiC}$ have long-lived electronic spin-triplet ground states that can be polarized and detected using light $[12,15,16]$, similar to NV centers in diamond. However, the divacancies have several potential advantages, particularly in the context of photonics. Namely, they are optically active near the telecom wavelengths, and their host material, $\mathrm{SiC}$, is available commercially as lowimpurity, single-crystal wafers up to several inches in diameter, and is amenable to many existing device fabrication protocols $[17,18]$. In addition, 3C-SiC can be grown as an epitaxial film on $\mathrm{Si}$, which provides a natural way to obtain thin membranes for photonics and micromechanics applications rather than the thinning of bulk crystals [19-22]. Incorporating good spin qubits into photonic structures has been a long-standing area of interest $[23,24]$, and this has partly driven the substantial amount of research in studying the spin and optical properties of $\mathrm{SiC}$ divacancy ensembles over the past few years [12,25-27]. Recently, single divacancies with millisecond spin coherence times were isolated in $4 \mathrm{H}-\mathrm{SiC}$, which allows the control of single quantum states and the emission of single photons [28]. If the divacancy's spin state could be coherently mapped to photons, divacancy spins separated by long distances could be coherently linked via optical fiber $[1,29,30]$. Such a spin-photon interface would require highly spin-selective optical transitions, but only weakly spin-dependent optical readout has been demonstrated in $\mathrm{SiC}$ defects thus far [28,31]. Furthermore, achieving practical photon-mediated entanglement rates in defects, including the divacancy, will likely require integration with photonic structures that improve their spectral indistinguishability and photon emission rate. The general device friendliness of SiC may ease the fabrication of these structures [32], but no single defect spins have been isolated specifically in $3 \mathrm{C}-\mathrm{SiC}$, where thin membranes are easiest to create.

\section{ISOLATION OF SINGLE DIVACANCIES IN 3C-SiC}

Divacancies in $\mathrm{SiC}$ are a point defect consisting of a missing $\mathrm{Si}$ atom adjacent to a missing $\mathrm{C}$ atom. $\mathrm{SiC}$ may exist in a variety of different polytypes, but the most commonly studied and readily available polytypes are labeled $4 \mathrm{H}-$, $6 \mathrm{H}-$, and $3 \mathrm{C}-\mathrm{SiC}$. These polytypes have different stacking periodicity and crystalline structure, and, as a result, a different number of inequivalent divacancies exist in each polytype. There is a single form of divacancy in 3C-SiC, four inequivalent forms in $4 \mathrm{H}-\mathrm{SiC}$, and six in $6 \mathrm{H}-\mathrm{SiC}$. Ensembles of each inequivalent divacancy form in its neutral charge state have been detected using optically detected magnetic resonance (ODMR) in $4 \mathrm{H}-$ and $6 \mathrm{H}-\mathrm{SiC}$ using their distinct ground-state spin resonances [33].

Although $3 \mathrm{C}$ has thus far received less attention for coherent spin control than $4 \mathrm{H}$ and $6 \mathrm{H}$, it may be a very important polytype for photonics and optomechanical applications because it can be grown as an epitaxial thin film on $\mathrm{Si}$ [21,34-36]. An electron paramagnetic resonance (EPR) defect labeled "Ky5" [37] and the ODMR center labeled "L3" [38] in 3C-SiC have similar magnetic and optical properties to the predicted $3 \mathrm{C}-\mathrm{SiC}$ neutral divacancy [39] and those observed in the hexagonal polytypes [33], but only tentative assignment as the neutral divacancy has been made.

To isolate single Ky5/L3 defects, we start with a thick $(\sim 1.5 \mathrm{~mm})$ single crystal and polytype-inclusion-free bulklike layer of 3C-SiC grown by sublimation on $4 \mathrm{H}-\mathrm{SiC}$ substrate. This layer has significantly better epitaxial matching and lower density of structural defects compared to 3C-SiC material grown on $\mathrm{Si}$ substrates [40]. After removing the $4 \mathrm{H}-\mathrm{SiC}$ substrate and the defective interface region by mechanical polishing, we obtain a $730-\mu$ m-thick freestanding $3 \mathrm{C}-\mathrm{SiC}$ layer. In order to create a low density of Ky5/L3 defects, we irradiate the sample with $2.5 \mathrm{MeV}$ electrons at room temperature to a fluence of $5 \times 10^{12} \mathrm{~cm}^{-2}$ and perform a 30-min anneal in $\mathrm{Ar}$ gas at $745^{\circ} \mathrm{C}$ (see Supplemental Material [41]). Spatially resolved PL scans using off-resonant 975-nm laser excitation show isolated bright spots [Fig. 1(a)] with saturation count rates of about $26 \mathrm{kcts} / \mathrm{s}$ that have a zerophonon line at $1106 \mathrm{~nm}$ (see Supplemental Material [41]), which is consistent with previous Ky 5 and L 3 reports $[33,38]$. Photon coincidence correlation measurements [Fig. 1(b)] on several of these spots show an antibunching signal consistent with an isolated quantum emitter [42], which proves that they are from single defects.

We measure continuous-wave ODMR on these single defects by sweeping an applied microwave frequency and monitoring the PL emitted under off-resonant laser excitation. The spin resonances we detect are consistent with a spin-1 defect with a zero-field splitting (ZFS) of $D=$ $1.336 \mathrm{GHz}$. This is in agreement with previous Ky5 reports and shows that the L3 defect that has been detected in ODMR and the Ky5 defect that has been detected in EPR are the same defect. To demonstrate coherent control of these single spins, we detect coherent Rabi oscillations by initializing and detecting the spin using $\sim 1-\mu$ s laser pulses and applying microwave bursts between pulses for spin rotation [Fig. 1(c)]. The $7.5 \%$ Rabi readout contrast we 


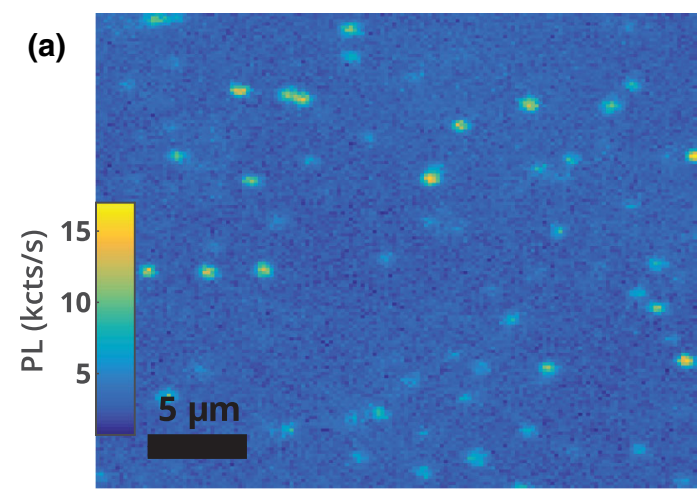

(c)

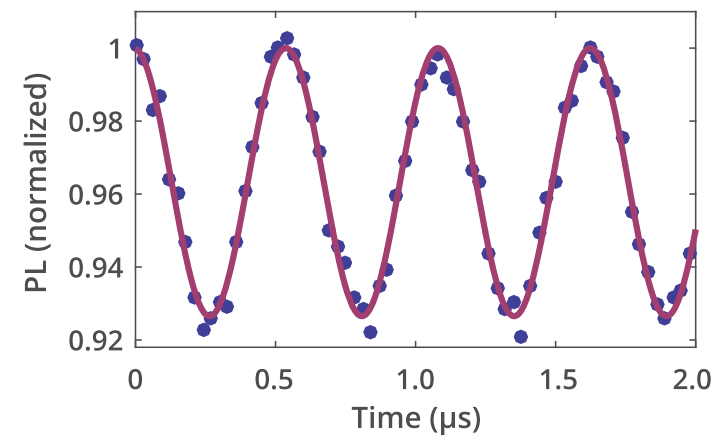

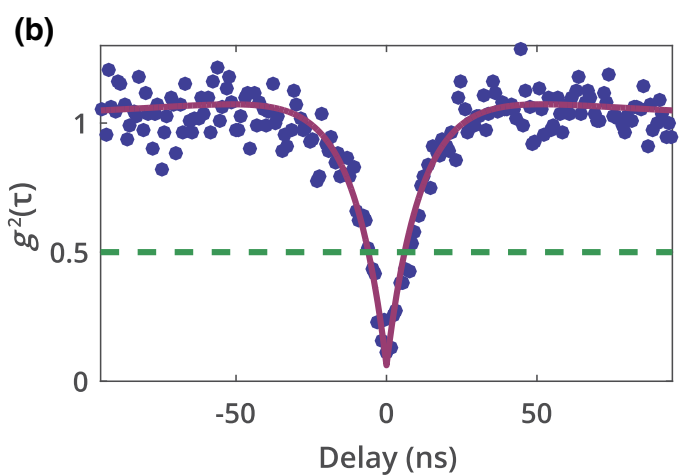

(d)

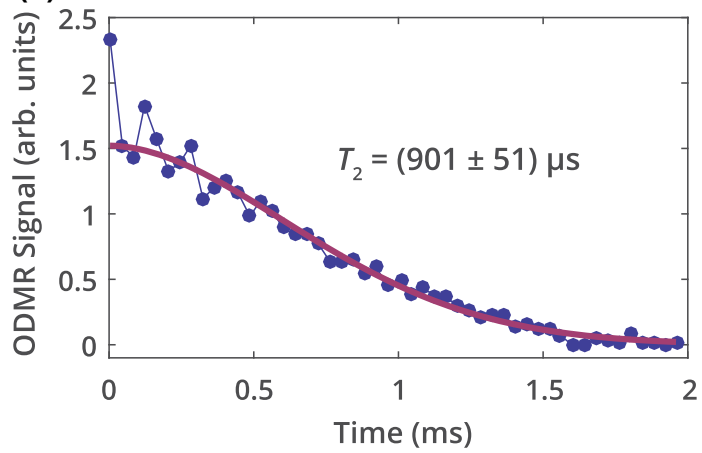

FIG. 1. Isolation and control of single spins in 3C-SiC. (a) A typical two-dimensional photoluminescence scan showing single defects as isolated luminescent spots in 3C-SiC. (b) Photon antibunching observed in the background-corrected photon correlation measurement taken on an isolated photoluminescence spot. The green dashed line indicates the 0.5 threshold below which the emission is consistent with a single quantum emitter. Our analysis indicates $g^{2}(\tau=0)=\left(0.055_{-0.047}^{+0.044}\right)$ at the $95 \%$ probability level, which confirms we have isolated a single defect. (c) A measurement of Rabi oscillations recorded on the same defect, which shows an approximate off-resonant readout contrast of 7.5\%. (d) Hahn-echo measurement performed on an ensemble of Ky5/L3 defects in the higher-irradiation $3 \mathrm{C}$-SiC sample $\left(1 \times 10^{15} \mathrm{~cm}^{-2}\right.$ fluence $)$ at $T=20 \mathrm{~K}$ and $B=253 \mathrm{G}$, showing $T_{2}=(901 \pm 51) \mu \mathrm{s}$.

measure, defined as the absolute change in PL intensity divided by the off-resonant PL intensity, is similar to the $9 \%-15 \%$ contrasts seen in different forms of single $4 \mathrm{H}-\mathrm{SiC}$ divacancies [28].

We characterize the inhomogeneous spin coherence times $\left(T_{2}^{*}\right)$ of a few of these defects using Ramsey spectroscopy. The longest we observe is $T_{2}^{*}=(1.8 \pm 0.1) \mu \mathrm{s}$, and the shortest is $(250 \pm 90) \mathrm{ns}$ on a different defect (see Supplemental Material [41]). Differences in the local impurity density may explain this variation. To probe their homogeneous coherence time, we apply a Hahn-echo pulse sequence on a second sample that contains an ensemble of Ky5/L3 defects created with a large $1 \times 10^{15} \mathrm{~cm}^{-2}$ irradiation fluence. This measurement sets a lower bound on the single-spin homogeneous coherence time, and the larger signal makes such a long-duty-cycle measurement more practical. We observe $T_{2}=(0.90 \pm 0.05) \mathrm{ms}$ at $T=20 \mathrm{~K}$ and $B=253 \mathrm{G}$ [Fig. 1(d)]. These coherence times are substantially longer than both those in previous reports in Ky5 ensembles $\left(T_{2}^{*}=52 \mathrm{~ns}\right.$ and $T_{2}=24 \mu \mathrm{s}$ ) [33], longer than those seen in nonisotopically purified diamond NV center ensembles $\left(T_{2}=0.63 \mathrm{~ms}\right)$ [43], and comparable to those of $4 \mathrm{H}-\mathrm{SiC}$ divacancies $\left(T_{2}=1.3 \mathrm{~ms}\right)[28,44]$. It is likely that this coherence time can be improved to several milliseconds in isotopically purified $\mathrm{SiC}$ or by using more sophisticated dynamical decoupling sequences $[44,45]$.

To identify Ky5/L3, we compare ab initio density functional theory simulations of the $3 \mathrm{C}-\mathrm{SiC}$ neutral divacancy to experimental reconstructions of the Ky5/L3 hyperfine coupling tensors [46]. We use a 512-atom simulation of $3 \mathrm{C}-\mathrm{SiC}$ in VASP to compute the principal axes of the neutral divacancy's hyperfine coupling to ${ }^{29} \mathrm{Si}$ nuclei in the two inequivalent nearest-neighbor Si lattice sites, $\mathrm{Si}_{I I a}$ and $\mathrm{Si}_{I I b}$, which are threefold and sixfold degenerate, respectively, and to ${ }^{13} \mathrm{C}$ nuclei at the nearest-neighbor $\mathrm{C}$ lattice sites, $\mathrm{C}_{\mathrm{I}}$, which are threefold degenerate (Table I). These simulations use the projector augmented wave approach with HSE06 functionals [47-49], which we expect to be accurate to within $20 \%$ or better (an error less than $1.5 \mathrm{MHz}$ for ${ }^{29} \mathrm{Si}_{\mathrm{II} a}$ and ${ }^{29} \mathrm{Si}_{\mathrm{II} b}$ ). Single spins have less inhomogeneity, and hence tend to have larger $T_{2}^{*}$ coherence times, than ensembles. This increases the spectral resolution of our pulsed ODMR measurements, and we are able to detect shifts of less than $1 \mathrm{MHz}$.

We locate two single Ky5/L3 defects where we observe low-field hyperfine splittings of 59 and $9 \mathrm{MHz}$ in pulsed 
TABLE I. Density functional theory simulations of the neutral 3C-SiC divacancy's hyperfine tensors for ${ }^{13} \mathrm{C}$ and ${ }^{29} \mathrm{Si}$ nuclei, and our reconstructions from experimental data obtained from Ky5/L3 defects. The experimental values in parentheses are inferred from a global fit to pulsed ODMR measurements taken on single Ky5/L3 defects that are strongly coupled to a nearby nuclear spin, and are reported in parentheses with $95 \%$ credible intervals. The large uncertainty on $\theta$ for ${ }^{29} \mathrm{Si}_{\text {II } a}$ is a consequence of the fact that the tensor is nearly isotropic within the MHz resolution of our experiment.

\begin{tabular}{lccccc}
\hline \hline Nucleus site & $A_{x x}(\mathrm{MHz})$ & $A_{y y}(\mathrm{MHz})$ & $A_{z z}(\mathrm{MHz})$ & $\Theta(\mathrm{deg})$ & $A_{z}(\mathrm{MHz})$ \\
\hline${ }^{13} \mathrm{C}_{\mathrm{I}}$ & $51.3(49.5 \pm 4.5)$ & $52.0(49.5 \pm 4.5)$ & $122.2(108.5 \pm 3.6)$ & $72.6(72.3 \pm 4.3)$ & $61.0(57.6 \pm 1.3)$ \\
${ }^{29} \mathrm{Si}_{\text {II } a}$ & $9.1(8.7 \pm 1.0)$ & $9.9(8.7 \pm 1.0)$ & $7.7(9.5 \pm 1.0)$ & $68.5(47 \pm 34)$ & $8.9(9.1 \pm 0.2)$ \\
${ }^{29} \mathrm{Si}_{\text {II } b}$ & 11.4 & 11.4 & 11.8 & 50.1 & $11.6(12.4 \pm 1.2)$ \\
\hline \hline
\end{tabular}

ODMR measurements, which are near the expected hyperfine resonances (approximately $A_{z}$ ) for ${ }^{13} \mathrm{C}_{\mathrm{I}}$ and ${ }^{29} \mathrm{Si}_{\mathrm{II} b}$ nuclei. On each defect, we repeat pulsed ODMR measurements across both $m_{s}= \pm 1$ branches at different magnetic field strengths and angles $\left(B=10-250 \mathrm{G}, \theta=0^{\circ}-80^{\circ}\right)$. The varied fields hybridize the electronic and nuclear spin states in slightly different ways, which manifest as small shifts in the hyperfine resonance locations. From a global fit to these ODMR scans, we infer the principal values of the hyperfine tensors, $A_{x x}, A_{y y}$, and $A_{z z}$, and the angle $\theta$ between the principal $z$ axis of the tensor and the defect's symmetry axis in Table I, which are found by diagonalization [50]. The uncertainties of the fit indicate we are not sensitive to $A_{y y}$, so we set $A_{y y}=A_{x x}$ as an approximation. We also infer the $A_{z}$ value for the ${ }^{29} \mathrm{Si}_{\text {II } a}$ site from the previously described ensemble Hahn-echo measurement's electron spin echo envelope modulations (see Supplemental Material [41]). Besides the hyperfine tensors, our computations also predict a ground-state ZFS of $D=$ $1.32 \mathrm{GHz}$ for the $3 \mathrm{C}$ neutral divacancy, which is consistent with the $1.336-\mathrm{GHz}$ value we measure here to about the same accuracy as $a b$ initio calculations in the $4 \mathrm{H}$ and $6 \mathrm{H}$ polytypes [25]. The excellent quantitative agreements between our experiments and the simulated hyperfine couplings and ZFSs are very strong evidence that Ky5/ L3 is the neutral divacancy.

\section{RESONANT EXCITATION OF SiC DIVACANCIES}

Initialization and detection of the divacancy's groundstate spin using off-resonant excitation relies on the intrinsic transition rates of its optical cycle. The modest spin selectivity of this process means that emitted PL is only weakly spin-dependent, which manifests as the low contrast in Fig. 1(c), and that only a limited spin polarization can be generated. Resonant manipulation of divacancies via their optical fine structure may enable initialization and readout protocols of much higher fidelity because individual transitions, which may be highly spindependent, can be selectively addressed. Because of solidstate strain inhomogeneity, observing the optical fine structure directly requires resonant excitation of a single defect, not an ensemble. We have thus far isolated divacancies in $3 \mathrm{C}-\mathrm{SiC}$, and to extend our studies to $4 \mathrm{H}-\mathrm{SiC}$, we prepare a sample from the same wafer as the previous $4 \mathrm{H}-\mathrm{SiC}$ study where single divacancies were isolated [28,51]. The single divacancy in 3C-SiC and the $(h h)$ and $(k k)$ forms of divacancy in $4 \mathrm{H}-\mathrm{SiC}$ should each have a ${ }^{3} E$ excited state because of their $\mathrm{C}_{3 v}$ symmetry and because each has six active electrons. We focus on these forms because a detailed theoretical treatment of this electronic structure, developed in the context of diamond NV centers, is already available in two recent works $[52,53]$.

The various interactions in the ${ }^{3} E$ excited-state manifold play a critical role in the high-fidelity control of divacancies using resonance fluorescence techniques. In Fig. 2(a), we diagram the six ${ }^{3} E$ excited state sublevels' responses to spin-orbit $\left(\lambda_{z}\right)$, spin-spin $\left(D_{\mathrm{es}}, \Delta_{1}, \Delta_{2}\right)$, and transverse strain $\left(\delta_{\perp}\right)$ interactions. The effect of $\lambda_{z}, D_{\mathrm{es}}$, and $\Delta_{1}$ is to split or shift certain sublevels, while the effect of $\Delta_{2}$ is to mix the $E_{1}$ and $E_{2}$ states with the $E_{x}$ and $E_{y}$ states. These pairs of states have differing spin character, but at values of $\delta_{\perp}$ that are low relative to $\lambda_{z}$, and if $\Delta_{2}$ is small, mixing between them will be low, and the $E_{x}$ and $E_{y}$ levels will retain an almost purely $m_{s}=0$ character. In this case, transitions to the $E_{x}$ or $E_{y}$ levels from the $m_{s}=0$ groundstate sublevel are cycling transitions since they have a very high probability of returning to the initial $m_{s}=0$ state via spontaneous emission. The fidelity of spin readout using such cycling transitions can be quite high because the transition has both a high spin-selectivity and the ability to be cycled repeatedly in order to extract multiple photons per measurement shot. This counteracts the low collection efficiencies of real-world experiments, and if the degree of spin mixing is low enough, can even allow single-shot detection [54-56]. Such highly spin-dependent transitions can also be used to generate spin-photon entanglement, which is an essential precursor for remote entanglement mediated by photons [57]. These functionalities will be significantly degraded by even a small amount of mixing within the excited-state manifold, so a precise determination the intrinsic spin-orbit and spin-spin interactions is crucial for determining the ultimate preparation and readout fidelities obtainable in $\mathrm{SiC}$ divacancies. 

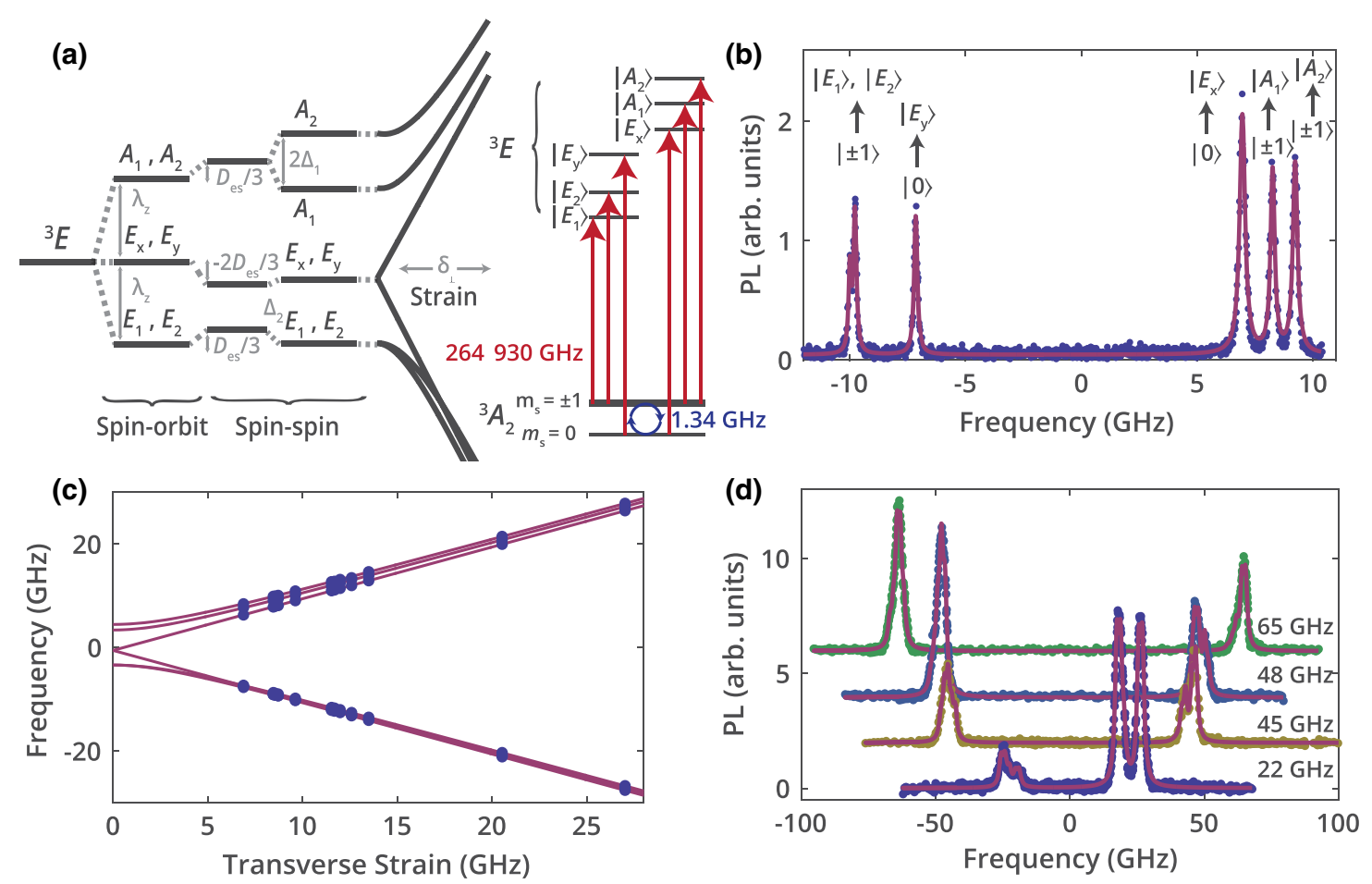

FIG. 2. Excited-state fine structure of $4 \mathrm{H}-$ and $3 \mathrm{C}-\mathrm{SiC}$ divacancies. (a) Left: Level diagram of the ${ }^{3} E$ excited state, showing the effect of axial spin-orbit coupling, the three spin-spin interactions, and transverse strain. Right: Diagram of optical transitions excited in a PLE measurement that are nominally spin-conserving at low strain, which are near $264930 \mathrm{GHz}$ for $(h h)$ divacancies. Microwave driving near $D=1.34 \mathrm{GHz}$ is applied to produce a mixed time-averaged population in the ground state so that all six transitions can be observed. (b) Photoluminescence excitation measurements taken on a single $4 \mathrm{H}-\mathrm{SiC}(h h)$ divacancy with a transverse strain of $\delta_{\perp}=$ $7 \mathrm{GHz}$ at $T=8 \mathrm{~K}$ under continuous microwave driving. (c) Individual resonances recorded at $T=8 \mathrm{~K}$ on separate $(h h)$ divacancies that experience different magnitudes of transverse strain. The solid lines are the Hamiltonian energies at the best fit value. (d) Photoluminescence excitation measurements taken $T=8 \mathrm{~K}$ on single $3 \mathrm{C}$-SiC divacancies that experience different transverse strains. The estimated magnitude of the transverse strain is labeled in units of $\mathrm{GHz}$ on each trace. The solid lines are fits to a sum of Lorentzians intended only as a guide to the eye since the larger linewidths make the number of spin-conserving and spin-nonconserving transitions that are visible uncertain.

To create single divacancies in $4 \mathrm{H}-\mathrm{SiC}$, we irradiate our sample with $2-\mathrm{MeV}$ electrons at room temperature to a fluence of $5 \times 10^{12} \mathrm{~cm}^{-2}$, and anneal it at $745^{\circ} \mathrm{C}$ in Ar gas for $30 \mathrm{~min}$. Single divacancies are easily seen in spatial PL scans, and have saturation PL intensities of about $27 \mathrm{kcts} / \mathrm{s}$ in our apparatus (see Supplemental Material [41]). At temperatures below $T=20 \mathrm{~K}$, transitions between different ground- and excited-state sublevels become sharp and we can observe the optical fine structure in a photoluminescence excitation (PLE) measurement. In this measurement, an off-resonant laser pulse first polarizes the ground-state spin into $m_{s}=0$, and we detect the sideband PL as we scan the frequency of a tunable narrow-line laser. Optionally, we may apply continuous microwaves to the $m_{s}= \pm 1$ magnetic resonances in the ground state so that transitions from those ground-state sublevels can also be observed. Figure 2(b) shows the PLE spectrum of a single 4H-SiC $(k k)$ divacancy measured at $T=8 \mathrm{~K}$, in which each of the six spin-allowed transitions are seen. Individual divacancies experience a randomly distributed strain, which manifests itself as approximately half of the separation between the $E_{x}$ and $E_{y}$ transitions, and is sample dependent. If no microwaves are applied, only the $E_{x}$ and $E_{y}$ transitions are seen (not shown), which implies that off-resonant light polarizes the spin to a high degree into $m_{s}=0$. This is also consistent with the strong polarization we observe in single 3C-SiC divacancies using time-resolved PL measurements (see Appendix).

The strength of each interaction term in the excited-state Hamiltonian can be inferred by analyzing the relative PLE resonance frequencies as a function of transverse strain [58]. We perform PLE measurements on $13(h h)$ and $10(k k)$ divacancies, which we find at randomly distributed transverse strains, and use a Bayesian analysis (see Supplemental Material [41]) to infer the spin-orbit and spin-spin interaction parameters intrinsic to both forms and the transverse strain of each particular defect. The data for the $(k k)$ divacancy and the model fit are shown in Fig. 2(c). A similar plot for $(h h)$ divacancies, along with raw PLE data taken on a single basally oriented $(k h)$ divacancy, can be found in the Supplemental Material [41]. The agreement of the data and model for both $(h h)$ and $(k k)$ 
forms is generally within the $10-\mathrm{MHz}$ repeatability of our wave meter. For the $(h h)$ divacancies, which have zero phonon lines near $264.91 \mathrm{THz}$, we infer $\lambda_{z}=(3.538 \pm$ $0.052) \mathrm{GHz}, D_{\mathrm{es}}=(0.855 \pm 0.017) \mathrm{GHz}, \Delta_{1}=(0.577 \pm$ $0.019) \mathrm{GHz}$, and $\Delta_{2}=\left(0.031_{-0.031}^{+0.050}\right) \mathrm{GHz}$, while for the $(k k)$ divacancies, which have zero phonon lines near $265.31 \mathrm{THz}$, we infer $\lambda_{z}=(6.090 \pm 0.052) \mathrm{GHz}, D_{\mathrm{es}}=$ $(0.852 \pm 0.012) \mathrm{GHz}, \quad \Delta_{1}=(0.584 \pm 0.012) \mathrm{GHz}$, and $\Delta_{2}=\left(0.044_{-0.044}^{+0.046}\right) \mathrm{GHz}$, where all values are quoted with a $95 \%$ credible interval. As mentioned, the same general ${ }^{3} E$ structure is also present in NV centers, but the specific interaction strengths of $\mathrm{SiC}$ divacancies mean that spin mixing in the $E_{x}$ level is about an order of magnitude weaker. This is mostly a consequence of having a weaker mixing term $\Delta_{2} \approx 40 \mathrm{MHz}$ versus about $200 \mathrm{MHz}$ in the $\mathrm{NV}$, while still retaining a comparable spin-orbit strength (3.5-6 GHz versus $5.3 \mathrm{GHz}$ ) [58]. If spin mixing is the main source of spin flips, then the expected number of photons a divacancy can emit is about $10^{4}$, compared to about $10^{3}$ in the NV center (see Supplemental Material [41]). However, at such low levels of mixing, other as-yet unquantified effects like residual coupling to the spinsinglet states or indirect spin flips from phonon-induced orbital transitions may become the most significant source of spin flips that limit the divacancy's emission [59]. Nevertheless, this is a promising prediction since low numbers of detected photons are a main limitation to the fidelity of current NV-based single-shot readout efforts. In addition, ionization and spectral hopping are further hindrances in NV experiments, and we have never observed either effect in our experiments on divacancies (see Supplemental Material [41]) [1,56,60]. The divacancy's intrinsic ability to emit at least as many photons per shot and to remain in the correct charge and resonance states over time will be a boon to single-shot readout and faster entanglement success rates in SiC.

To investigate the $3 \mathrm{C}-\mathrm{SiC}$ divacancy, we perform the same PLE measurement on single divacancies at $T=8 \mathrm{~K}$ near $270.95 \mathrm{THz}$. Figure 2(d) shows the PLE resonances of different single 3C divacancies with $\delta_{\perp}=22-65 \mathrm{GHz}$. The splitting of the two excited state branches with strain follows the characteristic behavior expected for the ${ }^{3} E$ excited state, and we infer $\lambda_{z}=(15.7 \pm 2.5) \mathrm{GHz}$, $D_{e s}=(2.0 \pm 1.0) \mathrm{GHz}$, and $\Delta_{1}, \Delta_{2}<1.0 \mathrm{GHz}$ for the $3 \mathrm{C}-\mathrm{SiC}$ divacancy's excited state. The linewidths we observe in this sample are nearly $2 \mathrm{GHz}$, which are significantly larger than the typical $100-200 \mathrm{MHz}$ linewidths we observe in divacancies in our $4 \mathrm{H}-\mathrm{SiC}$ sample. Given these linewidths, we cannot precisely infer the weaker spin-spin interaction terms, $\Delta_{1}$ and $\Delta_{2}$, which are critical for predicting spin-mixing behavior. We note, however, that the spin-orbit interaction is significantly stronger here, and if the values of $\Delta_{1}$ and $\Delta_{2}$ are similar to what we observe in the two forms of $4 \mathrm{H}-\mathrm{SiC}$ divacancy, mixing should be suppressed in $3 \mathrm{C}-\mathrm{SiC}$ by an additional order of magnitude compared to $4 \mathrm{H}-\mathrm{SiC}$. One additional peculiarity of this sample is that the PLE peak intensities change only negligibly when microwaves are applied. We might observe this behavior for an unpolarized spin with short spin coherence, but the off-resonant ODMR contrast being comparable to divacancies in $4 \mathrm{H}-\mathrm{SiC}$, the long Hahn-echo spin coherence time we measure, and the high polarization we infer from time-resolved PL (see Appendix) all suggest this is not the case. We surmise that a lower impurity sample will eliminate this behavior and will display linewidths comparable to $4 \mathrm{H}-\mathrm{SiC}$.

An optical transition's linewidth should be as narrow as possible so that two-photon interference can be observed, which is necessary for remote entanglement $[61,62]$. We observe typical linewidths of 100-200 MHz in our 4H-SiC sample, with a particular $(k k)$ divacancy showing transitions as narrow as $80 \mathrm{MHz}$ at $T=5 \mathrm{~K}$. This is promising since these initial values are already comparable to the 36-483 MHz linewidths of NV centers used in spin-photon and spin-spin entanglement experiments [62-64]. The divacancy's lifetime-limited linewidth, however, is $11 \mathrm{MHz}$, and it is important to establish whether the broadenings we observe in $4 \mathrm{H}-\mathrm{SiC}$ and especially $3 \mathrm{C}-\mathrm{SiC}$ are due to intrinsic interactions with lattice phonons or by impurities that can potentially be reduced in future efforts. Impurities in solids, including diamond, often broaden optical linewidths via temperature-independent charge fluctuations [65-67]. To investigate, we measure the optical linewidths of each form of divacancy in both samples as a function of temperature [Fig. 3(a)]. At temperatures above $T=20 \mathrm{~K}$, the linewidth of each form follows a $T^{5}$ dependence $[68,69]$, while at lower temperatures, the linewidths of each form approach an asymptote. Notably, at the lowest temperatures, the linewidths for different inequivalent divacancies in our $4 \mathrm{H}-\mathrm{SiC}$ sample each approach about $100 \mathrm{MHz}$, while the linewidth of the divacancy measured in our 3C-SiC sample remains near $2 \mathrm{GHz}$. We suspect that the larger linewidths in our 3C$\mathrm{SiC}$ sample originate from its significantly higher nitrogen concentration of $8 \times 10^{15} \mathrm{~cm}^{-3}$, versus the $5 \times 10^{13} \mathrm{~cm}^{-3}$ nitrogen concentration in our $4 \mathrm{H}-\mathrm{SiC}$ sample. The identical linewidths among inequivalent forms in the same $4 \mathrm{H}-\mathrm{SiC}$ sample, similar broadening at high temperatures, temperature independence below $20 \mathrm{~K}$, and prior reports of poor linewidths in nitrogen-rich diamond, are all consistent with the idea that the linewidth broadening we observe at low temperatures originates from sample-dependent impurities, rather than intrinsic interactions with phonons [65,69-72]. These results suggest that reducing impurities and annealing any residual crystal damage are promising routes towards achieving linewidths closer to lifetime-limited in $\mathrm{SiC}$ divacancies $[73,74]$.

We have thus far probed the excited-state structure of divacancies in $\mathrm{SiC}$, and our measurements predict that it can be used for high-fidelity polarization and readout because of the low degree of intrinsic spin-mixing in the ${ }^{3} E$ state. We now show explicitly that certain optical 

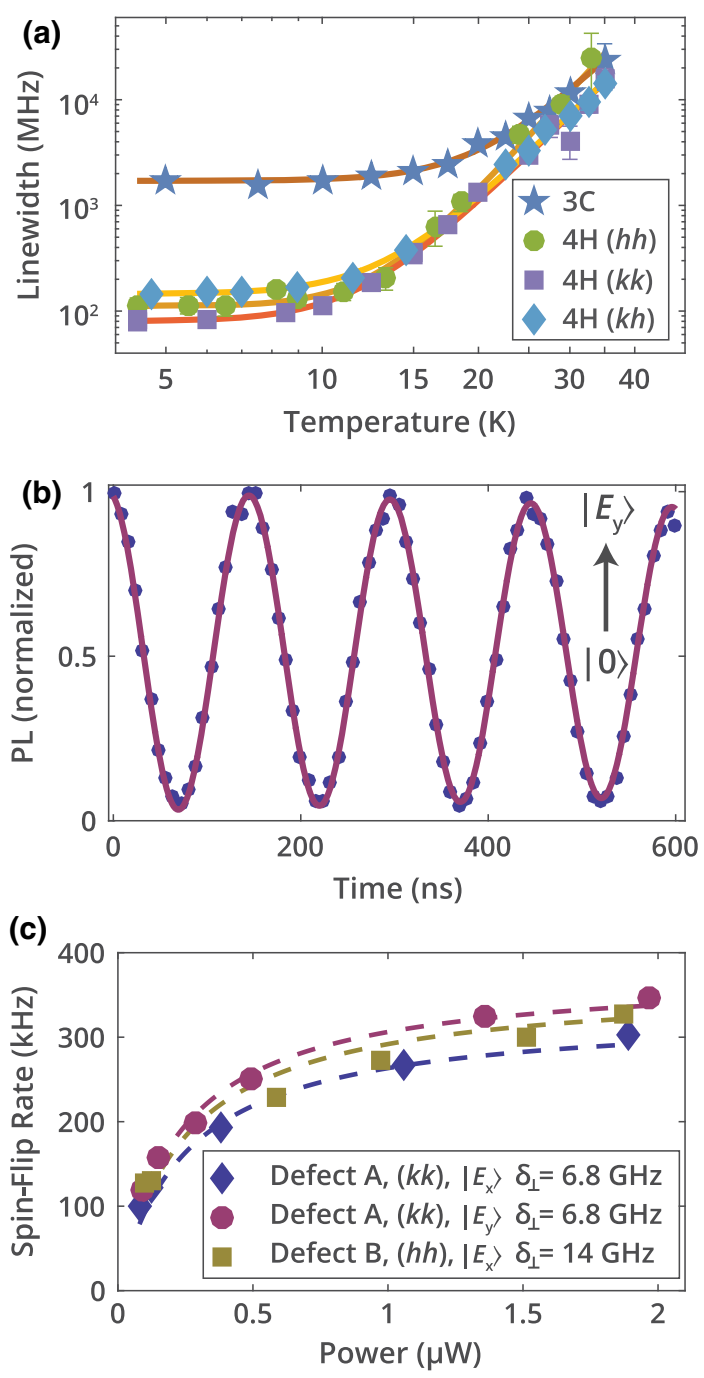

FIG. 3. Characterization of divacancy optical transition properties. (a) Temperature dependence of the optical linewidths of different inequivalent $3 \mathrm{C}-\mathrm{SiC}$ and $4 \mathrm{H}-\mathrm{SiC}$ divacancy defects. (b) Single-spin Rabi oscillations recorded using the $E_{y}$ transition of a single $4 \mathrm{H}-\mathrm{SiC}(h h)$ divacancy at $T=8 \mathrm{~K}$ and $B=15 \mathrm{G}$ with a transverse strain of $\delta_{\perp}=27 \mathrm{GHz}$. The measurement gives a background-corrected readout contrast of $94 \%$, consistent with a high degree of spin polarization. (c) The spin-flip rate as a function of resonant laser power measured on different $E_{x}$ and $E_{y}$ transitions on different single $4 \mathrm{H}-\mathrm{SiC}$ divacancies.

transitions are highly spin-dependent, and that they can be used for high-contrast detection of the ground-state spin. The Rabi oscillations of a single $4 \mathrm{H}-\mathrm{SiC}(h h)$ divacancy detected via resonant excitation of its $E_{y}$ transition are shown in Fig. 3(b). In this measurement, we apply an off-resonant laser pulse to prepare the spin, and a variablelength microwave burst for spin rotation [as in Fig. 1(c)], but the spin is detected by applying a resonant laser pulse and monitoring the sideband PL. While standard off-resonant readout gives only a 9\% contrast [28], the measurement here has a $94 \%$ readout contrast. This significant improvement originates from both the high ground-state polarization induced by the divacancy's optical cycle (consistent with the Appendix) and because the spin mixing of $E_{y}$ at this divacancy's transverse strain of $\delta_{\perp}=27 \mathrm{GHz}$ is not excessive. Imperfect ground-state spin polarization is now likely to be the limiting factor in this measurement's contrast, and it can be improved in the future by using a second laser to deplete the residual $m_{s}= \pm 1$ population via driving of the $E_{1}, E_{2}, A_{1}$, or $A_{2}$ transitions. In addition, we expect that using ultrapure epilayers without removing the substrate will produce lower strain $\left(\delta_{\perp}<3 \mathrm{GHz}\right)$ divacancies where superior spin-mixing behavior will increase the transition's spin selectivity and the number of detected photons per measurement.

The fidelity of single-shot readout of $\mathrm{SiC}$ divacancies will depend sensitively on the number of photons detected per measurement shot, and the ultimate fidelities that can be obtained in practice will depend on both the total number of photons emitted and the efficiency by which they can be collected. Improved collection efficiencies were recently demonstrated in $\mathrm{SiC}$ defects [31], and our earlier analysis predicts that the $(h h)$ and $(k k)$ divacancies should have a low spin-flip probability and, as a consequence, emit at least as many photons per shot as the NV center. However, as we also discuss, other as-yet unquantified effects like residual coupling to the spin-singlet states could increase the rate of spin flips actually realized in experiment, which would degrade the total number of photons emitted per shot. To measure the spin-flip rate, we apply an offresonant pulse to polarize a single divacancy's spin, and then time resolve the photons emitted when we drive an $E_{x}$ or $E_{y}$ transition. As the spin is repeatedly excited, the PL decays exponentially in time as the spin has more chances to flip, and we report the reciprocal of the decay time as the spin-flip rate. Figure 3(b) shows the spin-flip rates of a few divacancies, which increase with laser power because the spin has more chances to flip per unit time, and eventually saturate because of the divacancies' finite optical lifetime. The saturation rates we measure are near $330 \mathrm{kHz}$, and within the $150-400 \mathrm{kHz}$ saturation rates of $\mathrm{NV}$ centers already used for high-fidelity single-shot readout [56]. Since the optical lifetimes of $(h h)$ and $(k k)$ divacancies are similar to the NV center [25,75], the similar rate of spin flips at saturation suggests the total number of photons emitted should also be roughly similar to NVs. We note further that the divacancies we measure here experience suboptimal strain, which causes an increased spin-flip rate due to excited-state mixing. According to our model of the excited state, spin mixing at this particular defect's transverse strain of $\delta_{\perp}=6.8 \mathrm{GHz}$ is roughly 4 times higher than the ideal value near $2 \mathrm{GHz}$, and, hence, that improvements in material strain should allow for even lower spin-flip rates to be realized in $\mathrm{SiC}$. If the same photon collection efficiencies realized in diamond can be translated to divacancies in $\mathrm{SiC}$, it appears that spin flips should be sufficiently low to achieve single-shot readout of $\mathrm{SiC}$ divacancies with the same or even potentially higher fidelities. 


\section{CONCLUSION}

We perform PL, PLE, and magnetic resonance experiments on single $3 \mathrm{C}$ - and $4 \mathrm{H}-\mathrm{SiC}$ divacancies aimed at revealing fundamental aspects of their electronic structure and their optical and spin properties. We find that the divacancy in $3 \mathrm{C}-\mathrm{SiC}$ can be isolated at the single defect limit and has a Hahn-echo electronic spin coherence time that is nearly $1 \mathrm{~ms}$ long. This is an exciting result since $3 \mathrm{C}-\mathrm{SiC}$ can be heteroepitaxially grown as a thin film on $\mathrm{Si}$, which provides a natural way to obtain suspended membranes that can be processed into photonic cavities. In both $3 \mathrm{C}-\mathrm{SiC}$ and $4 \mathrm{H}-\mathrm{SiC}$, we reveal a highly spin-dependent optical fine structure that provides a pathway to highfidelity initialization and single-shot readout, which are necessary for remote entanglement protocols. This interface has favorable spin-mixing properties, and the optical linewidths we observe in $4 \mathrm{H}-\mathrm{SiC}$ are already near those required for photon-mediated entanglement. Our results also highlight the importance that materials efforts play in driving the control of quantum states in semiconductors in that low-impurity and low-strain material are needed for narrow optical transitions and ideal spin-mixing properties in both $3 \mathrm{C}-\mathrm{SiC}$ and $4 \mathrm{H}-\mathrm{SiC}$. Since the divacancies in $\mathrm{SiC}$ operate near the telecom wavelengths and possess a highly spin-selective spin-photon interface, we expect that our findings here will stimulate research into using them as building blocks in future quantum networks.

\section{ACKNOWLEDGEMENTS}

We are grateful to Paolo Andrich for assistance in sample preparation, and Abram L. Falk and Brian B. Zhou for helpful discussions. This work was funded by ARO W911NF-15-2-0058, AFOSR FA9550-15-1-0029, and FA9550-14-1-0231, NSF MRSEC DMR-1420709, the DOE LDRD Program, Swedish Research Council (6212014-5825 and 2016-04068), ÅForsk foundation (16-576), Carl-Trygger Stiftelse för Vetenskaplig Forskning (CTS 15:339), Knut and Alice Wallenberg Foundation (KAW 2013.0300), JSPS KAKENHI B 26286047, and Swedish Energy Agency (43611-1).

\section{APPENDIX: RATE EQUATION MODELING OF 3C-SiC DIVACANCY OPTICAL CYCLE}

It is empirically clear that off-resonant cycling of divacancies can both polarize and detect their electronic spin states [12,15], but prior works have not focused on the details of the divacancy's optical cycle that underlie these functionalities. Based on their common $\mathrm{C}_{3 v}$ symmetry and six active electrons, the $(h h)$ and $(k k)$ divacancies in $4 \mathrm{H}-\mathrm{SiC}$, the divacancy in $3 \mathrm{C}-\mathrm{SiC}$, and the nitrogen-vacancy center in diamond should share the same electronic orbital levels but with different relative energy positions and different transition rates between levels. Besides the ${ }^{3} A_{2}$ ground state and the ${ }^{3} E$ excited state, which we focus on in the main text, the ${ }^{1} A_{1}$ and ${ }^{1} E$ spin-singlet levels also exist $[11,76]$. The specific transition rates between orbital and spin levels are responsible for how much spin polarization can be generated, the speed of the polarization and readout process, and the change in PL when the spin populates an $m_{s}= \pm 1$ sublevel versus $m_{s}=0$. These are important fundamental questions for future experiments, and we can use time-resolved PL experiments on single spins to establish a simplified model of the 3C-SiC divacancy's optical cycle.

We propose a five-level model of the divacancy's spin and orbital levels in Fig. 4(a). In this model, the ground and excited states are treated as orbital singlets with one level corresponding to $m_{s}=0$ and another corresponding to the $m_{s}= \pm 1$ within each orbital. A fifth level represents one or more spin-singlet states, and transitions into and out of this level are treated as nonradiative. For the divacancy's spin to both polarize under repeated optical excitation and generate a change in fluorescence based on its spin state, at least one transition should be spin-selective and nonradiative. One possible mechanism, in analogy with the NV center, is that the $m_{s}= \pm 1$ sublevels of the ${ }^{3} E$ state undergo a nonradiative intersystem crossing (ISC) to a spin-singlet state via a nonaxial spin-orbit interaction [54,77].

To explicitly probe spin-dependent decay pathways from the excited state, we time resolve the PL emitted when a short laser pulse is applied after the ground-state spin is prepared in $m_{s}=0$ and $m_{s}= \pm 1$. In this experiment, a $1-\mu \mathrm{s}-$ long $975-\mathrm{nm}$ off-resonant pulse polarizes the spin into $m_{s}=0$, a $1-\mu$ s delay allows the defect to relax back to its ground state, and a subnanosecond $920-\mathrm{nm}$ pulse from a mode-locked Ti:sapphire laser excites the spin $[75,78,79]$. The PL induced by this last pulse is time-resolved in Fig. 4(b) when no microwaves are applied and when a $\pi$ pulse on the $m_{s}=-1$ transition is applied just before the subnanosecond optical pulse. The PL follows a biexponential decay in both traces, and the relative amplitudes of the two lifetime components track the proportion of $m_{s}=0$ and $m_{s}= \pm 1$ at intermediate spin rotation angles [80,81]. The shorter lifetime is indicative of a nonradiative decay out of the excited state, and the dependence on the groundstate spin indicates it is spin-selective. This result is analogous to similar experiments in NV centers, and is solid evidence that their optical cycles function in a similar way [75]. The solid lines are fits we perform on both data sets simultaneously (see Supplemental Material [41]) and reveal the two lifetimes as $\tau_{0}=(18.7 \pm 0.3) \mathrm{ns}$ and $\tau_{1}=(15.7 \pm 0.3) \mathrm{ns}$. The difference in the two lifetimes suggests that the excited-state-to-singlet-transition rates differ by $(10 \pm 2) \mathrm{MHz}$, depending on whether the spin is in $m_{s}= \pm 1$ or $m_{s}=0$. This measurement also indicates that the ground-state spin polarization is quite high. By computing the relative fraction of the two lifetime components, we infer a $96_{-5}^{+4} \%$ spin polarization in the ground state. This is close to the high polarization required for a $94 \%$ Rabi oscillation contrast observed in a $4 \mathrm{H}-\mathrm{SiC}$ 

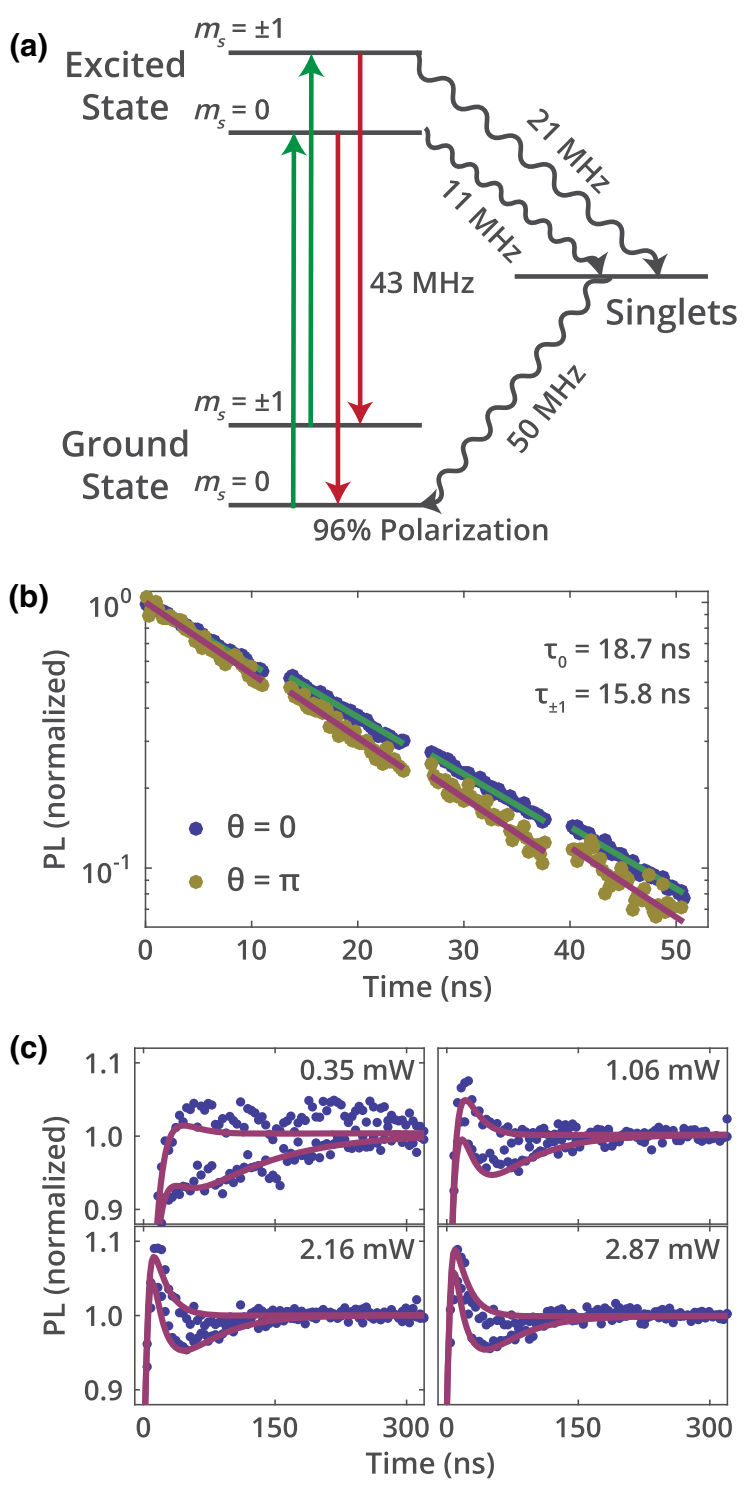

FIG. 4. Dynamical model of the 3C-SiC divacancy. (a) Diagram of the levels and major rates in the five-level rate-equation model. The transition rates and ground-state spin polarization are inferred from the global fit described in the main text. (b) Normalized log plot of the fluorescence recorded on a single 3C-SiC divacancy. The $\theta=0$ data are the time-resolved PL when no microwaves are applied and the spin remains in $m_{s}=0$, while the $\theta=\pi$ data are taken after a microwave $\pi$ pulse prepares the spin in the $m_{s}=-1$ state. The small gaps originate from a preprocessing step where small pulses imperfectly extinguished by our electro-optic modulator are removed. (c) Normalized single divacancy photoluminescence emitted at the beginning off-resonant cw laser pulses of different powers. The upper trace in each subpanel is the PL observed when the spin is prepared in $m_{s}=0$, while the respective lower traces are observed when a microwave $\pi$ pulse rotates the spin into $m_{s}=-1$. Solid lines are the corresponding global fits of the rate-equation model.

divacancy in Fig. 3(b), which illustrates another similarity between divacancies in different polytypes.

To infer the remaining rates, we perform a second experiment where we prepare the spin in either $m_{s}=0$ or $m_{s}=-1$ using the same cw laser pulse and microwave pulses as the previous experiment, but time-resolve the PL emitted under a second cw laser pulse. The PL dynamics under continuous excitation are more complicated than biexponential decay since the PL no longer depends on only a subset of the defect's transition rates. To control for overfitting, we perform the experiment at 11 laser powers between 0.35 and $2.87 \mathrm{~mW}$ and perform a fit of the rateequation model to all 11 pairs of data sets simultaneously. This excitation range covers below and above the intensity at which the defect's PL saturates (see Supplemental Material [41]). During the fitting process, the ISC rates and polarization are constrained by the biexponential decay experiment, and the relative optical pumping strengths are fixed based on the measured laser intensities. Allowing for decay from the singlet states to $m_{s}= \pm 1$ levels in the ground state does not improve the fit significantly, so this term is set to zero. Four experimental runs where the spin is prepared in either $m_{s}=0$ or $m_{s}=-1$ are shown in Fig. 4(d), where the solid lines in each subplot are the output of the global fitting procedure.

The amount of similarity between the orbital and spinselective dynamics of $\mathrm{SiC}$ divacancies and diamond $\mathrm{NV}$ centers has been an open question, and our model's quantitative reproduction of the observed PL dynamics is good evidence that the special optical cycle seen in NV centers is also present in $\mathrm{SiC}$ divacancies. The biexponential decay experiment explicitly shows that a spin-selective and nonradiative transition exists out of the excited state, which is likely the reason why the divacancy's spin can be polarized and detected using off-resonant laser light. This transition is only weakly spin-selective, however, and is about 7 times slower than the corresponding NV center rate. The singlet decay rate we infer for divacancies is also about 20 times faster than that of NV centers [75], and consistent with recent qualitative ensemble measurements [82]. Together, this means that a spin in the $m_{s}= \pm 1$ state will undergo a nonradiative transition less often, and that when it does, it quickly decays to the ground state where it can be excited again and emit photons. These observations likely explain the weaker $7.5 \%$ off-resonant readout contrast [Fig. 1(c)] of 3C-SiC divacancies relative to the nearly $30 \%$ seen in NV centers, and may also explain the 9\%-15\% contrasts seen in single $4 \mathrm{H}-\mathrm{SiC}$ divacancies [28]. We also find that allowing the $m_{s}=0$ level in the excited state to undergo an ISC to the singlet noticeably improves the quality of the fit, but a transverse spin-orbit interaction should only couple to states with $m_{s}= \pm 1$ spin [59]. This may be partially explained by this sample's high strain, since it will partially mix the excited-state sublevels and thus violate our model's assumption of sublevels with pure spin character. As we mention, the fit is constrained by the biexponential decay experiment, so the model suggests the optical lifetime of $23 \mathrm{~ns}$ is shortened to an apparent value of $18.7 \mathrm{~ns}$ because of this additional nonradiative ISC decay. 
In the future, measuring all six excited-state branching ratios using resonant spectroscopy on a low-strain sample will give the clearest insight into the details of the divacancy's ISC [59].

[1] H. Bernien, B. Hensen, W. Pfaff, G. Koolstra, M. S. Blok, L. Robledo, T. H. Taminiau, M. Markham, D. J. Twitchen, L. Childress, and R. Hanson, Heralded Entanglement between Solid-State Qubits Separated by Three Metres, Nature (London) 497, 86 (2013).

[2] W. Pfaff, B. J. Hensen, H. Bernien, S. B. van Dam, M. S. Blok, T. H. Taminiau, M. J. Tiggelman, R. N. Schouten, M. Markham, D. J. Twitchen, and R. Hanson, Unconditional Quantum Teleportation between Distant Solid-State Quantum Bits, Science 345, 532 (2014).

[3] B. Hensen, H. Bernien, A. E. Dréau, A. Reiserer, N. Kalb, M. S. Blok, J. Ruitenberg, R. F. L. Vermeulen, R. N. Schouten, C. Abellán, W. Amaya, V. Pruneri, M. W. Mitchell, M. Markham, D. J. Twitchen, D. Elkouss, S. Wehner, T. H. Taminiau, and R. Hanson, Loophole-Free Bell Inequality Violation Using Electron Spins Separated by 1.3 Kilometres, Nature (London) 526, 682 (2015).

[4] L. J. Rogers, K. D. Jahnke, M. H. Metsch, A. Sipahigil, J. M. Binder, T. Teraji, H. Sumiya, J. Isoya, M. D. Lukin, P. Hemmer, and F. Jelezko, All-Optical Initialization, Readout, and Coherent Preparation of Single Silicon-Vacancy Spins in Diamond, Phys. Rev. Lett. 113, 263602 (2014).

[5] J. R. Rabeau, Y. L. Chin, S. Prawer, F. Jelezko, T. Gaebel, and J. Wrachtrup, Fabrication of Single Nickel-Nitrogen Defects in Diamond by Chemical Vapor Deposition, Appl. Phys. Lett. 86, 131926 (2005).

[6] R. Kolesov, K. Xia, R. Reuter, R. Stöhr, A. Zappe, J. Meijer, P. R. Hemmer, and J. Wrachtrup, Optical Detection of a Single Rare-Earth Ion in a Crystal, Nat. Commun. 3, 1029 (2012).

[7] P. Siyushev, K. Xia, R. Reuter, M. Jamali, N. Yang, C. Duan, N. Kukharchyk, A. D. Wieck, J. Wrachtrup, N. Zhao, and R. Kolesov, Coherent Properties of Single Rare-Earth Spin Qubits, Nat. Commun. 5, 3895 (2014).

[8] W. F. Koehl, B. Diler, S. J. Whiteley, A. Bourassa, N. T. Son, E. Janzén, and D. D. Awschalom, Resonant Optical Spectroscopy and Coherent Control of $\mathrm{Cr}^{4+}$ Spin Ensembles in SiC and GaN, Phys. Rev. B 95, 035207 (2017).

[9] J. R. Weber, W. F. Koehl, J. B. Varley, A. Janotti, B. B. Buckley, C. G. Van de Walle, and D. D. Awschalom, Quantum Computing with Defects, Proc. Natl. Acad. Sci. U.S.A. 107, 8513 (2010).

[10] P. G. Baranov, A. P. Bundakova, A. A. Soltamova, S. B. Orlinskii, I. V. Borovykh, R. Zondervan, R. Verberk, and J. Schmidt, Silicon Vacancy in SiC as a Promising Quantum System for Single-Defect and Single-Photon Spectroscopy, Phys. Rev. B 83, 125203 (2011).

[11] A. Gali, Time-Dependent Density Functional Study on the Excitation Spectrum of Point Defects in Semiconductors, Phys. Status Solidi 248, 1337 (2011).

[12] W. F. Koehl, B. B. Buckley, F. J. Heremans, G. Calusine, and D. D. Awschalom, Room Temperature Coherent
Control of Defect Spin Qubits in Silicon Carbide, Nature (London) 479, 84 (2011).

[13] H. Kraus, V. a. Soltamov, D. Riedel, S. Väth, F. Fuchs, A. Sperlich, P. G. Baranov, V. Dyakonov, and G. V. Astakhov, Room-Temperature Quantum Microwave Emitters Based on Spin Defects in Silicon Carbide, Nat. Phys. 10, 157 (2013).

[14] S. Castelletto, B. C. Johnson, V. Ivády, N. Stavrias, T. Umeda, A. Gali, and T. Ohshima, A Silicon Carbide Room-Temperature Single-Photon Source, Nat. Mater. 13, 151 (2013).

[15] P. G. Baranov, I. V. Il'in, E. N. Mokhov, M. V. Muzafarova, S. B. Orlinskii, and J. Schmidt, EPR Identification of the Triplet Ground State and Photoinduced Population Inversion for a Si-C Divacancy in Silicon Carbide, J. Exp. Theor. Phys. Lett. 82, 441 (2005).

[16] N. T. Son, P. Carlsson, J. ul Hassan, E. Janzén, T. Umeda, J. Isoya, A. Gali, M. Bockstedte, N. Morishita, T. Ohshima, and H. Itoh, Divacancy in 4H-SiC, Phys. Rev. Lett. 96, 055501 (2006).

[17] S. E. Saddow and A. Agarwal, Advances in Silicon Carbide Processing and Applications (Artech House Publishers, London, 2004).

[18] A. Powell, J. Sumakeris, Y. Khlebnikov, M. Paisley, R. Leonard, E. Deyneka, S. Gangwal, J. Ambati, V. Tsevtkov, J. Seaman, A. McClure, J. Guo, M. Dudly, E. Balkas, and V. Balakrishna, Bulk Growth of Large Area SiC Crystals, Mater. Sci. Forum 858, 5 (2016).

[19] C. A. Zorman, A. J. Fleischman, A. S. Dewa, M. Mehregany, C. Jacob, S. Nishino, and P. Pirouz, Epitaxial Growth of 3C-SiC Films on 4 in. Diam (100) Silicon Wafers by Atmospheric Pressure Chemical Vapor Deposition, J. Appl. Phys. 78, 5136 (1995).

[20] Y. T. Yang, K. L. Ekinci, X. M. H. Huang, L. M. Schiavone, M. L. Roukes, C. A. Zorman, and M. Mehregany, Monocrystalline Silicon Carbide Nanoelectromechanical Systems, Appl. Phys. Lett. 78, 162 (2001).

[21] G. Calusine, A. Politi, and D. D. Awschalom, Silicon Carbide Photonic Crystal Cavities with Integrated Color Centers, Appl. Phys. Lett. 105, 011123 (2014).

[22] J. C. Lee, A. P. Magyar, D. O. Bracher, I. Aharonovich, and E. L. Hu, Fabrication of Thin Diamond Membranes for Photonic Applications, Diam. Relat. Mater. 33, 45 (2013).

[23] J. L. O'Brien, A. Furusawa, and J. Vučković, Photonic Quantum Technologies, Nat. Photonics 3, 687 (2009).

[24] I. Aharonovich, A. D. Greentree, and S. Prawer, Diamond Photonics, Nat. Photonics 5, 397 (2011).

[25] A. L. Falk, P. V. Klimov, B. B. Buckley, V. Ivády, I. A. Abrikosov, G. Calusine, W. F. Koehl, Á. Gali, and D. D. Awschalom, Electrically and Mechanically Tunable Electron Spins in Silicon Carbide Color Centers, Phys. Rev. Lett. 112, 187601 (2014).

[26] P. V. Klimov, A. L. Falk, B. B. Buckley, and D. D. Awschalom, Electrically Driven Spin Resonance in Silicon Carbide Color Centers, Phys. Rev. Lett. 112, 087601 (2014).

[27] O. V. Zwier, D. O'Shea, A. R. Onur, and C. H. van der Wal, All-Optical Coherent Population Trapping with Defect Spin Ensembles in Silicon Carbide, Sci. Rep. 5, 10931 (2015).

[28] D. J. Christle, A. L. Falk, P. Andrich, P. V. Klimov, J. Ul Hassan, N. T. Son, E. Janzén, T. Ohshima, and 
D. D. Awschalom, Isolated Electron Spins in Silicon Carbide with Millisecond Coherence Times, Nat. Mater. 14, 160 (2015).

[29] H. J. Kimble, The Quantum Internet, Nature (London) 453, 1023 (2008).

[30] W. B. Gao, A. Imamoglu, H. Bernien, and R. Hanson, Coherent Manipulation, Measurement and Entanglement of Individual Solid-State Spins Using Optical Fields, Nat. Photonics 9, 363 (2015).

[31] M. Widmann, S.-Y. Lee, T. Rendler, N. T. Son, H. Fedder, S. Paik, L.-P. Yang, N. Zhao, S. Yang, I. Booker, A. Denisenko, M. Jamali, S. A. Momenzadeh, I. Gerhardt, T. Ohshima, A. Gali, E. Janzén, and J. Wrachtrup, Coherent Control of Single Spins in Silicon Carbide at Room Temperature, Nat. Mater. 14, 164 (2014).

[32] J. Y. Lee, X. Lu, and Q. Lin, High-Q Silicon Carbide Photonic-Crystal Cavities, Appl. Phys. Lett. 106, 041106 (2015).

[33] A. L. Falk, B. B. Buckley, G. Calusine, W. F. Koehl, V. V. Dobrovitski, A. Politi, C. A. Zorman, P. X.-L. Feng, D. D. Awschalom, V. Viatcheslav, A. Politi, C. A. Zorman, X. Feng, and D. D. Awschalom, Polytype Control of Spin Qubits in Silicon Carbide, Nat. Commun. 4, 1819 (2013).

[34] J. Cardenas, M. Zhang, C. T. Phare, S. Y. Shah, C. B. Poitras, B. Guha, and M. Lipson, High Q SiC Microresonators, Opt. Express 21, 16882 (2013).

[35] M. Radulaski, T. M. Babinec, K. Müller, K. G. Lagoudakis, J. L. Zhang, S. Buckley, Y. A. Kelaita, K. Alassaad, G. Ferro, and J. Vučković, Visible Photoluminescence from Cubic (3C) Silicon Carbide Microdisks Coupled to High Quality Whispering Gallery Modes, ACS Photonics 2, 14 (2015).

[36] B.-S. Song, S. Yamada, T. Asano, and S. Noda, Demonstration of Two-Dimensional Photonic Crystals Based on Silicon Carbide, Opt. Express 19, 11084 (2011).

[37] V. Y. Bratus, R. S. Melnik, S. M. Okulov, V. N. Rodionov, B. D. Shanina, and M. I. Smoliy, A New Spin One Defect in Cubic SiC, Physica A (Amsterdam) 404B, 4739 (2009).

[38] N. T. Son, E. Sörman, W. M. Chen, C. Hallin, O. Kordina, B. Monemar, E. Janzén, and J. L. Lindström, Optically Detected Magnetic Resonance Studies of Defects in Electron-Irradiated 3C SiC Layers, Phys. Rev. B 55, 2863 (1997).

[39] L. Gordon, A. Janotti, and C. G. Van de Walle, Defects as Qubits in 3C- and 4H-SiC, Phys. Rev. B 92, 045208 (2015).

[40] V. Jokubavicius, G. R. Yazdi, R. Liljedahl, I. G. Ivanov, J. Sun, X. Liu, P. Schuh, M. Wilhelm, P. Wellmann, R. Yakimova, and M. Syväjärvi, Single Domain 3C-SiC Growth on Off-Oriented 4H-SiC Substrates, Cryst. Growth Des. 15, 2940 (2015).

[41] See Supplemental Material at http://link.aps.org/ supplemental/10.1103/PhysRevX.7.021046 for additional characterization data, analysis details, and PLE measurements on a basal-oriented divacancy.

[42] H. J. Kimble, M. Dagenais, and L. Mandel, Multiatom and Transit-Time Effects on Photon-Correlation Measurements in Resonance Fluorescence, Phys. Rev. A 18, 201 (1978).

[43] P. L. Stanwix, L. M. Pham, J. R. Maze, D. Le Sage, T. K. Yeung, P. Cappellaro, P. R. Hemmer, A. Yacoby, M. D. Lukin, and R.L. Walsworth, Coherence of Nitrogen-Vacancy Electronic Spin Ensembles in Diamond, Phys. Rev. B 82, 201201 (2010).

[44] H. Seo, A. L. Falk, P. V. Klimov, K. C. Miao, G. Galli, and D. D. Awschalom, Quantum Decoherence Dynamics of Divacancy Spins in Silicon Carbide, Nat. Commun. 7, 12935 (2016).

[45] L. P. Yang, C. Burk, M. Widmann, S. Y. Lee, J. Wrachtrup, and N. Zhao, Electron Spin Decoherence in Silicon Carbide Nuclear Spin Bath, Phys. Rev. B 90, 241203 (2014).

[46] K. Szasz, T. Hornos, M. Marsman, and A. Gali, Hyperfine Coupling of Point Defects in Semiconductors by Hybrid Density Functional Calculations: The Role of Core Spin Polarization, Phys. Rev. B 88, 075202 (2013).

[47] P. E. Blöchl, Projector Augmented-Wave Method, Phys. Rev. B 50, 17953 (1994).

[48] G. Kresse and J. Furthmüller, Efficient Iterative Schemes for Ab Initio Total-Energy Calculations Using a Plane-Wave Basis Set, Phys. Rev. B 54, 11169 (1996).

[49] J. P. Perdew, J. A. Chevary, S. H. Vosko, K. A. Jackson, M. R. Pederson, D. J. Singh, and C. Fiolhais, Atoms, Molecules, Solids, and Surfaces: Applications of the Generalized Gradient Approximation for Exchange and Correlation, Phys. Rev. B 46, 6671 (1992).

[50] V. Ivády, K. Szász, A. L. Falk, P. V. Klimov, D. J. Christle, E. Janzén, I. A. Abrikosov, D. D. Awschalom, and A. Gali, Theoretical Model of Dynamic Spin Polarization of Nuclei Coupled to Paramagnetic Point Defects in Diamond and Silicon Carbide, Phys. Rev. B 92, 115206 (2015).

[51] J. Hassan, J. P. Bergman, A. Henry, and E. Janzén, On-Axis Homoepitaxial Growth on Si-Face 4H-SiC Substrates, J. Cryst. Growth 310, 4424 (2008).

[52] J. R. Maze, A. Gali, E. Togan, Y. Chu, A. Trifonov, E. Kaxiras, and M. D. Lukin, Properties of Nitrogen-Vacancy Centers in Diamond: The Group Theoretic Approach, New J. Phys. 13, 025025 (2011).

[53] M. W. Doherty, N. B. Manson, P. Delaney, and L. C. L. Hollenberg, The Negatively Charged Nitrogen-Vacancy Centre in Diamond: The Electronic Solution, New J. Phys. 13, 025019 (2011).

[54] N. B. Manson, J. P. Harrison, and M. J. Sellars, NitrogenVacancy Center in Diamond: Model of the Electronic Structure and Associated Dynamics, Phys. Rev. B 74, 104303 (2006).

[55] P. Tamarat, N. B. Manson, J. P. Harrison, R. L. McMurtrie, A. Nizovtsev, C. Santori, R. G. Beausoleil, P. Neumann, T. Gaebel, F. Jelezko, P. Hemmer, and J. Wrachtrup, Spin-Flip and Spin-Conserving Optical Transitions of the NitrogenVacancy Centre in Diamond, New J. Phys. 10, 045004 (2008).

[56] L. Robledo, L. Childress, H. Bernien, B. Hensen, P. F. Alkemade, and R. Hanson, High-Fidelity Projective ReadOut of a Solid-State Spin Quantum Register, Nature (London) 477, 574 (2011).

[57] W. Pfaff, T.H. Taminiau, L. Robledo, H. Bernien, M. Markham, D. J. Twitchen, and R. Hanson, Demonstration of Entanglement-by-Measurement of Solid-State Qubits, Nat. Phys. 9, 29 (2013).

[58] A. Batalov, V. Jacques, F. Kaiser, P. Siyushev, P. Neumann, L. J. Rogers, R. L. McMurtrie, N. B. Manson, F. Jelezko, 
and J. Wrachtrup, Low Temperature Studies of the Excited-State Structure of Negatively Charged NitrogenVacancy Color Centers in Diamond, Phys. Rev. Lett. 102, 195506 (2009).

[59] M. L. Goldman, A. Sipahigil, M. W. Doherty, N. Y. Yao, S. D. Bennett, M. Markham, D. J. Twitchen, N. B. Manson, A. Kubanek, and M. D. Lukin, Phonon-Induced Population Dynamics and Intersystem Crossing in Nitrogen-Vacancy Centers, Phys. Rev. Lett. 114, 145502 (2015).

[60] V. M. Acosta, C. Santori, A. Faraon, Z. Huang, K. M. C. Fu, A. Stacey, D. A. Simpson, K. Ganesan, S. TomljenovicHanic, A. D. Greentree, S. Prawer, and R. G. Beausoleil, Dynamic Stabilization of the Optical Resonances of Single Nitrogen-Vacancy Centers in Diamond, Phys. Rev. Lett. 108, 206401 (2012).

[61] R. Lettow, Y. L. A. Rezus, A. Renn, G. Zumofen, E. Ikonen, S. Götzinger, and V. Sandoghdar, Quantum Interference of Tunably Indistinguishable Photons from Remote Organic Molecules, Phys. Rev. Lett. 104, 123605 (2010).

[62] H. Bernien, L. Childress, L. Robledo, M. Markham, D. Twitchen, and R. Hanson, Two-Photon Quantum Interference from Separate Nitrogen Vacancy Centers in Diamond, Phys. Rev. Lett. 108, 043604 (2012).

[63] E. Togan, Y. Chu, A. S. Trifonov, L. Jiang, J. Maze, L. Childress, M. V. G. Dutt, A. S. Sørensen, P. R. Hemmer, A.S. Zibrov, and M.D. Lukin, Quantum Entanglement between an Optical Photon and a Solid-State Spin Qubit, Nature (London) 466, 730 (2010).

[64] B. B. Buckley, G. D. Fuchs, L. C. Bassett, and D. D. Awschalom, Spin-Light Coherence for Single-Spin Measurement and Control in Diamond, Science 330, 1212 (2010).

[65] D. E. McCumber and M. D. Sturge, Linewidth and Temperature Shift of the R Lines in Ruby, J. Appl. Phys. 34, 1682 (1963).

[66] T. Basché, W. E. Moerner, M. Orrit, and U. P. Wild, SingleMolecule Optical Detection, Imaging and Spectroscopy (Verlag Chemie, Weinheim, 1997).

[67] J. Wolters, N. Sadzak, A. W. Schell, T. Schröder, and O. Benson, Measurement of the Ultrafast Spectral Diffusion of the Optical Transition of Nitrogen Vacancy Centers in Nano-Size Diamond Using Correlation Interferometry, Phys. Rev. Lett. 110, 027401 (2013).

[68] M. B. Walker, A T5 Spin-Lattice Relaxation Rate for NonKramers Ions, Can. J. Phys. 46, 1347 (1968).

[69] Kai-Mei C. Fu, C. Santori, P. E. Barclay, L. J. Rogers, N. B. Manson, and R. G. Beausoleil, Observation of the Dynamic Jahn-Teller Effect in the Excited States of Nitrogen-Vacancy Centers in Diamond, Phys. Rev. Lett. 103, 256404 (2009).

[70] D. A. Wiersma, Coherent Optical Transient Studies of Dephasing and Relaxation in Electronic Transitions of Large Molecules in the Condensed Phase, Adv. Chem. Phys. 47, 421 (1981).
[71] F. Jelezko, I. Popa, A. Gruber, C. Tietz, J. Wrachtrup, A. Nizovtsev, and S. Kilin, Single Spin States in a Defect Center Resolved by Optical Spectroscopy, Appl. Phys. Lett. 81, 2160 (2002).

[72] P. Tamarat, T. Gaebel, J. R. Rabeau, M. Khan, A. D. Greentree, H. Wilson, L. C. L. Hollenberg, S. Prawer, P. Hemmer, F. Jelezko, and J. Wrachtrup, Stark Shift Control of Single Optical Centers in Diamond, Phys. Rev. Lett. 97, 083002 (2006).

[73] Y. Chu, N. P. De Leon, B. J. Shields, B. Hausmann, R. Evans, E. Togan, M. J. Burek, M. Markham, A. Stacey, A. S. Zibrov, A. Yacoby, D. J. Twitchen, M. Loncar, H. Park, P. Maletinsky, and M. D. Lukin, Coherent Optical Transitions in Implanted Nitrogen Vacancy Centers, Nano Lett. 14, 1982 (2014).

[74] Y. Chen, P. S. Salter, S. Knauer, L. Weng, A. C. Frangeskou, C. J. Stephen, S. N. Ishmael, P. R. Dolan, S. Johnson, B. L. Green, G. W. Morley, M. E. Newton, J. G. Rarity, M. J. Booth, and J. M. Smith, Laser Writing of Coherent Colour Centres in Diamond, Nat. Photonics 11, 77 (2016).

[75] L. Robledo, H. Bernien, T. Van Der Sar, and R. Hanson, Spin Dynamics in the Optical Cycle of Single NitrogenVacancy Centres in Diamond, New J. Phys. 13, 025013 (2011).

[76] A. Gali, A. Gällström, N. T. Son, and E. Janzén, Theory of Neutral Divacancy in SiC: A Defect for Spintronics, Mater. Sci. Forum 645-648, 395 (2010).

[77] A. Lenef and S. C. Rand, Electronic Structure of the N-V Center in Diamond: Theory, Phys. Rev. B 53, 13441 (1996).

[78] D. M. Toyli, D. J. Christle, A. Alkauskas, B. B. Buckley, C. G. Van de Walle, and D. D. Awschalom, Measurement and Control of Single Nitrogen-Vacancy Center Spins Above 600 K, Phys. Rev. X 2, 031001 (2012).

[79] P. V. Klimov, A. L. Falk, D. J. Christle, V. V. Dobrovitski, and D. D. Awschalom, Quantum Entanglement at Ambient Conditions in a Macroscopic Solid-State Spin Ensemble, Sci. Adv. 1, e1501015 (2015).

[80] N. B. Manson and R. L. McMurtrie, Issues Concerning the Nitrogen-Vacancy Center in Diamond, J. Lumin. 127, 98 (2007).

[81] A. Batalov, C. Zierl, T. Gaebel, P. Neumann, I. Y. Chan, G. Balasubramanian, P. R. Hemmer, F. Jelezko, and J. Wrachtrup, Temporal Coherence of Photons Emitted by Single Nitrogen-Vacancy Defect Centers in Diamond Using Optical Rabi-Oscillations, Phys. Rev. Lett. 100, 077401 (2008).

[82] G. Calusine, A. Politi, and D. D. Awschalom, CavityEnhanced Measurements of Defect Spins in Silicon Carbide, Phys. Rev. Applied 6, 014019 (2016). 